\title{
Investigation into the mechanisms by which microwave heating enhances separation of water-in-oil emulsions
}

\author{
E.R. Binner*, J.P. Robinson, S.A. Silvester, S.W. Kingman, E.H. Lester
}

Process \& Environmental Research Division, Faculty of Engineering, University of Nottingham, Nottingham. NG7 2RD, UK.

*e-mail: eleanor.binner@nottingham.ac.uk; Tel: +44 (0)1159514080

\begin{abstract}
The separation of water-in-oil emulsions made with Azeri crude was investigated using natural gravity settling and microwave heating techniques. Separation times could be reduced by an order of magnitude compared with untreated emulsions. Increasing the salinity of the water phase leads to a $15 \%$ average decrease in the settling time for untreated emulsions compared with over $90 \%$ for microwave-heated emulsions. An image analysis technique showed that the observed increases in settling time could not be attributed to changes in viscosity alone. Significant coalescence of water droplets occurs during microwave heating, however the effects of coalescence and viscosity reduction cannot be completely decoupled. Despite this, it is clear that it is the thermal effect of microwave heating that leads to improvements in settling times, and that any advantages in microwave heating over conventional heating can
\end{abstract}


be explained by selective heating of the aqueous phase rather than so-called nonthermal effects.

\section{KEYWORDS}

Water-in-oil emulsion; microwave; coalescence; viscosity; interfacial tension; selective heating

\section{INTRODUCTION}

Emulsions arise during the production of oil downstream from the well. Water is present with the oil, particularly in the latter stages of the well life when water injection is used to enhance the production rate. On leaving the well the mixture, at high pressure, is let down through a choke valve so that it can be further processed at moderate pressures. The pressure reduction valve creates significant shear within the fluid and it is this pressure drop that causes the emulsions to form. The water must be separated before further downstream transport and processing can commence, and as the oil and water phases are immiscible gravity separation can be utilised to separate the two substances. Gravity separation occurs due to a density difference between oil and water, and the separation of the two phases is governed by the settling velocity, $u_{S}$. If $u_{S}$ is small then a large residence time is required for the separation to occur, hence separation vessels need to be very large or a number of separation modules need to be included. This can be prohibitive when the separation needs to be carried out on offshore platforms or in remote locations. 
The settling velocity can be increased by changing the properties of the emulsion system.

The settling velocity of water droplets can be increased by heating the water and surrounding oil. This reduces both the oil/water interfacial tension and viscosity of the oil $[1,2]$. Coalescence can be promoted by using chemical additives, which lower interfacial tension, or by applying a physical force to move the droplets together, which can be achieved using ultrasound or an electrostatic force [3]. There are several disadvantages with these methods;

1. Heating the whole process stream requires a significant amount of energy

2. Chemical demulsifiers can add a significant cost and environmental impact

3. Electrostatic Coalescers add significant cost, and are often limited to low water concentrations

One technique that has received attention from previous researchers is microwave heating. There are two main advantages of microwave heating over conventional heating of emulsions:

(i) The water phase of the emulsion can be heated selectively, thus reducing the overall energy requirements

(ii) Microwave heating is volumetric, meaning that the entire dispersed water phase is heated instantaneously, without the limitations of conductive and convective heat transfer. 
Many workers have shown that, via selective heating of the water, microwave heating is able to enhance the separation of water-in-oil emulsions. Fortuny et al [4] report coalescence of water droplets during microwave heating, and attribute this to the reduction of the interfacial tension of the emulsion. Fang, Lai and Chang [5] report similar findings, using zeta potential to characterise their emulsions. They showed a reduction in the zeta potential of an oil-water emulsion after microwave treatment, and attribute this to the "microwave induced molecular rotation neutralizing the zeta potential of emulsified oil droplets'. Xia et al. [6, 7] studied conventional and microwave assisted demulsification, and they too suggest a reduction in zeta potential due to microwave heating. They also report that higher temperatures were achieved in their microwave demulsification experiments compared to those carried out using conventional heating. Ferreira et al. [8] also compared conventional and microwave heating, and looked at the effects of aging, demulsifier dosage and addition time. They showed that microwave-heated emulsions separated faster than conventionallyheated emulsions that were heated to the same average bulk temperature. Fang and Lai [9] carried out batch microwave demulsification experiments using 50/50 and 70/30 oil/water emulsions made with motor oil, and Tan et al [10] report the use of chemical demulsifiers along with batch microwave heating. Lemos et al. [11] and Guzmán-Lucero et al. [12] investigated the addition of ionic liquids (IL) in conjunction with microwave treatment. Fortuny et al. [13] proposed a promising new method for measuring salinity in crude oils that involved a microwave demulsification step and Binner et al. [14] evaluated a continuous flow system to assess the impact of industrial processing conditions on microwave enhanced emulsion separation. 
A significant number of empirical studies have been carried out which prove the principle of microwave assisted demulsification of water-in-oil emulsions, and report the importance of $\mathrm{pH}$, temperature, water and electrolyte content. Fortuny et al. and Lemos et al. $[4,11]$ report a reduction in demulsification efficiency with the addition of electrolytes, but their experiments were carried out at constant temperature, which led to a reduction in microwave power input with the addition of $\mathrm{NaCl}$. On the other hand, Xia et al. [7] report that the addition of various salts enhanced conventional and microwave assisted demulsification. The addition of inorganic salts was said to improve demulsification by destroying the double charge layers and increasing the density of the aqueous phase. The microwave treatment was said to be enhanced by the addition of salts because they increased the dielectric properties of the aqueous phase. Comparisons between microwave and conventional heating have been attempted $[8,11,15,16]$ but are limited by the difficulty in measuring absorbed power, temperature and temperature distribution during microwave heating. These difficulties have led to the proposal that non-thermal microwave effects exist, which subsequently lead to a reduction in zeta-potential $[4,5,12,16]$, and that "the magnetic field formed by the microwave makes the non-polar oil molecules magnetise and form a spiral electric field that subtends an angle with the axis of the oil molecule" [7]. However, the rationale or physical basis for these effects is not fully explained and the exact mechanisms of microwave assisted demulsification are still not fully understood.

The aim of the work reported in this paper is to investigate these mechanisms with the hypothesis that the phenomena observed during microwave assisted emulsification can be explained by selective and volumetric heating and heat and mass transfer 
processes. This knowledge will enable comparison of microwave assisted demulsification with demulsification by conventional heating methods, which will be required to assess the commercial potential of this process.

\section{METHODOLOGY}

The crude oil used in this study was Azeri crude supplied by BP. The assay and physical properties can be found on the BP website [17]. The oil was supplied surfactant-free, and no surfactants were added during this study.

\subsection{Emulsion formation}

A known amount of water was added to the Azeri crude resulting in an emulsion with a total volume of $500 \mathrm{ml}$. For specified experiments, $\mathrm{NaCl}$ was added to the water phase at $15 \mathrm{~g} / \mathrm{l}$ and $35 \mathrm{~g} / \mathrm{l}$. Fluorescein was also added (2ppm in water) to improve the tracking of the separation interface during data analysis. The samples were backlit with a UV light to show the water (with fluorescein layer as it forms and grows). After this procedure, each mixture was homogenised using a double stirrer for even energy distribution. The stirring frequency and mixing duration were kept constant at $600 \mathrm{rpm}$ and 2 minutes respectively.

A high standard of emulsion reproducibility was confirmed by utilising optical microscopy techniques. A droplet of newly homogenised emulsion was analysed by placing a droplet on a clear glass slide. The slide was backlit and magnified 200x (20x objective and internal 10x lens) on an Ortholux Pol II BK microscope with a 1 Megapixel camera, and the size of water droplets in the crude phase analysed using a 
calibrated sizing program written with Kontron KS400 version 3.1 System. An example of the droplet size distribution obtained for a 70/30 oil/water emulsion is shown in Figure 1 [14].

\subsection{Microwave Heating \& Interface tracking}

Once prepared the emulsions, in a $600 \mathrm{ml}$ beaker, were immediately placed in a 2.45 GHz 800W continuous power output multimode microwave cavity (300x300x200 $\mathrm{mm})$, and heated for $60-140$ seconds. After heating the emulsion was filmed using a HD Sony HDR-FX7 camcorder. Individual frames from the digital video (minimum 1 frame per minute to a maximum of 24 frames per second) were sequentially analysed using a MATLAB program (using image analysis toolbox, 2007 Edition). By thresholding the light green from black, the height of the water/oil boundary could be quantified, thus enabling the oil-water interface to be automatically tracked, as it rose with time.

The temperature was not measured as microwave processing results in thermal gradients between the microwave absorbing (water) and transparent phases (oil). These gradients undoubtedly change with experimental conditions but, by definition, cannot be quantified using bulk temperature measurements.

The reproducibility of the demulsification tests using microwave irradiation was evaluated through the repetition of tests. One sample within each set of the separation experiments was repeated at least twice in order to assess the reproducibility of the test method. The maximum fluctuation in the separation time results, for a given set of 
experimental conditions, was found to be $<10 \%$. For a high proportion of the microwave enhanced separations, a variation of only $+/-3 \%$ was observed.

\section{RESULTS}

Overall, the behaviour of 12 emulsions of Azeri crude oil and water were investigated, and the compositions of these emulsions are shown in Table 1.

\begin{tabular}{|c|c|c|}
\hline Emulsion \# & $\begin{array}{c}\text { Aqueous Phase } \\
(\%)\end{array}$ & $\begin{array}{l}\mathrm{NaCl} \text { concentration } \\
\text { in aqueous phase } \\
(\mathrm{g} / \mathrm{L})\end{array}$ \\
\hline 1 & 30 & 0 \\
\hline 2 & 30 & 15 \\
\hline 3 & 30 & 35 \\
\hline 4 & 20 & 0 \\
\hline 5 & 20 & 15 \\
\hline 6 & 20 & 35 \\
\hline 7 & 10 & 0 \\
\hline 8 & 10 & 15 \\
\hline 9 & 10 & 35 \\
\hline 10 & 5 & 0 \\
\hline 11 & 5 & 15 \\
\hline 12 & 5 & 35 \\
\hline
\end{tabular}

Table 1. Composition of emulsions used in this study 
The interface height was monitored after completion of the microwave heating process, for different heating conditions. Figure 2 shows example data for a 70/30 emulsion under three different processing conditions.

It can be seen that the untreated emulsion takes much longer to separate than those subject to microwave heating, with the interface taking approximately 1 hour to reach its maximum height. The microwave heated emulsions can be separated in a much shorter time, with the maximum interface height reached in less than one-third of the time. Also of note in Figure 2 is that the initial rate of separation is much faster in the microwave heated emulsions than the untreated. The raw data shown in Figure 2 is used to calculate the time taken from the beginning of treatment to the time the interface reaches its maximum height. This separation time is used to compare the effect of water content and salt content. Figure 3 shows the separation time plotted against the heating time taken from the raw data as demonstrated in Figure 2.

\subsection{Separation Time vs Water Content}

The data in Figure 3 show that the separation times decrease with increasing heating time for all water contents from 5-30\%. Separation times converge when heating times were $>120$ seconds despite significant differences in the separation time for untreated emulsions. For example, the emulsion containing 5\% water took 10,000 seconds to separate whereas the 70/30 emulsion took 4000 seconds. The difference between the separation times for the four untreated emulsions is likely to arise from the different sizes of water droplets present in each emulsion. This results from the constant stirring speed and constant stirring time when making the emulsions. From 
an energy balance standpoint the energy imparted by the stirrer creates surface area within the emulsions due to the shear force created, the rest is lost as heat. The same stirring conditions are expected to yield the same total droplet surface area within the emulsion, so for a smaller water volume the droplet diameter must be smaller to give the same surface area as that for a larger water volume. The trends shown in Figure 3 follow this hypothesis; the 95/5 emulsion contains the smallest droplets and takes the longest to separate whereas the 70/30 emulsion will have the largest droplets and will separate in the shortest time.

Despite the differences in separation time of untreated emulsions, the data in Figure 3 show that the separation time of all four emulsions is comparable after $>120$ seconds of microwave heating. There are several possible mechanisms contributing to this behaviour:

1. The effect of temperature on viscosity. The microwaves heat the water, and heat is transferred from the water to the oil interfacial layer. The increased temperature causes the oil viscosity to decrease, which increases the settling velocity. The microwave power applied to the emulsions was the same in each case; however the water is heated selectively over the oil. If heat transfer is neglected, an energy balance means that the lower mass of water in the $95 / 5$ emulsion will attain a higher temperature than the larger mass of water in the 70/30 emulsion, but the initial interfacial area is the same. The decrease in viscosity, and hence increase in settling velocity, will therefore be more marked in the lower water content emulsion. 
2. The effect of temperature on interfacial tension (and hence coalescence). The interfacial tension is reduced as the temperature increases [2], and this promotes coalescence. Again, this effect is more marked in the low-water emulsions due to higher temperatures in the water phase.

3. Other temperature effects. The probability of coalescence is not only a function of interfacial tension, but also droplet size distribution, number of droplets and dispersion density. Since those parameters were not constant across the different starting emulsions, the effect of different temperature profiles on each emulsion is difficult to predict.

4. Effect of droplet size on dielectric properties. The droplet size has been shown to affect the dielectric properties of emulsions. Holtze et al. [3] reported a peak in dielectric properties for water-in-oil emulsions somewhere between 3.6 $\mu \mathrm{m}$ and $>10 \mu \mathrm{m}$ mean droplet diameter. Although more data would be required on the specific emulsions used in this study, it is possible that microwaves heat the lower water content emulsions more effectively due to their smaller droplet size.

\subsection{Separation Time vs Salt Content}

Before identifying the effects of salt content on microwave demulsification tests the effect of salinity on untreated emulsions was investigated. Figure 4 shows the separation time of four different emulsions made with pure water, $15 \mathrm{~g} / \mathrm{L}$ and $35 \mathrm{~g} / \mathrm{L}$ $\mathrm{NaCl}$. The addition of salt causes a decrease in separation time for the 80/20, 90/10 and $95 / 5 \mathrm{oil} /$ water emulsions, with the most significant decrease with the $95 / 5$ emulsions where the separation time was reduced from 10000 seconds to 7000 
seconds. The separation time of the 70/30 emulsion underwent a slight increase when $\mathrm{NaCl}$ was added. The addition of electrolytes is known to alter the interfacial tension of an emulsion [1], and the general effect is to improve the probability of coalescence. However, in this case the technique for emulsion manufacture was kept constant and it could be that the salinity affects the droplet size distribution. It is not possible, therefore, to completely decouple the effects of interfacial tension and droplet size in Figure 4. It does show, however, that with the exception of the $95 / 5$ oil/water emulsion, the addition of salt has little effect on the settling time of untreated emulsions.

The effect of $\mathrm{NaCl}$ content on the separation behaviour of the 70/30 emulsion after microwave treatment is shown in Figure 5. The addition of $15 \mathrm{~g} / \mathrm{L} \mathrm{NaCl}$ appears to make little difference to the separation of the emulsion compared with the emulsion made with pure water. However, when $35 \mathrm{~g} / \mathrm{L}$ is added the separation time is decreased from that of the emulsion made with pure water.

Figure 6 shows the effect of $\mathrm{NaCl}$ content on the separation behaviour of the 90/10 emulsion after microwave treatment. The addition of $\mathrm{NaCl}$ caused a reduction in separation time across the whole range of heating times tested, and similar results were also observed in the $80 / 20$ and $95 / 5$ emulsions.

There are several underlying physical mechanisms that combine to give the separation profiles shown in Figures 5 and 6. The addition of electrolytes reduces the interfacial tension between the oil and water phases and therefore increases the probability that adjacent droplets will coalesce to form larger droplets. Figure 4 shows that this effect 
is minimal for most of the untreated emulsions studied. However, interfacial tension also decreases with increasing temperature [2], so coalescence is promoted during microwave heating, and this effect may be enhanced by the presence of electrolytes.

The effect of salinity on the dielectric properties of the water phase, and hence its ability to be heated, is another factor. Increasing $\mathrm{NaCl}$ concentration introduces an ionic conduction component to the loss mechanism, leading to improved heating compared with pure water. The dielectric properties of pure and saline water are shown in Table 2.

\begin{tabular}{|c|c|c|}
\hline \multirow{2}{*}{$\begin{array}{c}\mathrm{NaCl} \\
\text { concentration }\end{array}$} & \multicolumn{2}{|c|}{$2.45 \mathrm{GHz}$} \\
\cline { 2 - 3 }$(\mathrm{g} / \mathrm{l})$ & $\varepsilon^{\prime}$ & $\varepsilon^{\prime \prime}$ \\
\hline 0 & 77 & 13 \\
\hline 15 & 70 & 17 \\
\hline 35 & 68 & 54 \\
\hline
\end{tabular}

Table 2. Dielectric properties of saline water [18]

The dielectric property data shown in Table 2 can be used to determine the relative electric field and power density distribution within the water phase of the emulsions. Exact determination of these parameters would require very sophisticated 3D electromagnetic simulations, using sub-micron mesh sizes to account for the size distribution of the water droplets in the emulsion. An alternative but equally valid approach is to establish the relative power dissipated within water droplets if the 
electric field strength at the interface is constant. Figure 7 shows the result of this approach.

The electric field decays as the distance from the interface increases, and the relative strength of the electric field does not differ significantly between pure water and the two salt concentrations used in this study. However, the power density changes dramatically due to the large change in dielectric loss factor at $35 \mathrm{~g} / \mathrm{l}$. In this case the power density at the interface is more than three times that of pure water, leading to higher interface temperatures and subsequently a reduced viscosity and reduced interfacial tension. The evidence from Figures 4 and 7 therefore leads to the conclusion that $\mathrm{NaCl}$ enhances the thermal effect during microwave heating, rather than directly changing the interfacial tension.

The combined effects of salt content and increased temperature due to microwave heating lead to a significant reduction in separation time with all the emulsions studied. The mechanisms by which enhanced separation occurs are droplet coalescence or a viscosity reduction in the oil film adjacent to the water droplet. The experimental settling data, coupled with measurements of the droplet size distribution shown in Figure can be used to estimate the extent to which coalescence occurs.

\subsection{Modelling the emulsion separation process}

For the water droplets at the top of the beaker of liquid to fall to the interface layer means they must travel a set distance, depending upon the water content used; for example water droplets in a 70/30 emulsion must travel a maximum distance of $5 \mathrm{~cm}$. 
At room temperature the minimum velocity of water droplet of a set diameter can be calculated using Stokes' Law and the physical properties of the oil [17], and the separation time calculated based on the time to travel $5 \mathrm{~cm}$ at that velocity. The separation times for a range of droplet sizes are shown in Figure 8.

From Figure 1 the mean diameter of water droplets in the emulsion is around $20 \mu \mathrm{m}$. The predicted separation time for droplets of this size is over $1 \times 10^{6}$ seconds, compared to experimental data of 4000 seconds. To achieve the separation times for the untreated emulsion, at room temperature, the average droplet diameter must be $345 \mu \mathrm{m}$, almost a two orders of magnitude increase from the starting average of $20 \mu \mathrm{m}$. The difference between the predicted and actual separation times shows that significant droplet coalescence must occur during the droplet settling process, even without microwave or conventional heating.

A second model was developed to assess the likely degree of coalescence during the microwave heating process. It is assumed that the average droplet size is $345 \mu \mathrm{m}$, i.e. the size of droplets due to coalescence in the untreated emulsion. The separation time of the $345 \mu \mathrm{m}$ droplets can be predicted based on the assumed temperature of the surrounding oil phase. It is known that the water phase is heated selectively over the oil, however heat transfer will occur during settling, which increases the temperature of the oil and reduces its viscosity. For a constant droplet size, Stokes' Law can be used to predict the separation time for a given oil viscosity, which can be related to temperature according to the physical property data [17]. Figure 9 shows the predicted separation time for microwave heated emulsions based on an oil temperature of $100^{\circ} \mathrm{C}$, along with the minimum observed experimental settling time. 
The maximum temperature that can be achieved in the oil phase during the microwave heating experiments is $100^{\circ} \mathrm{C}$, the boiling point of water at atmospheric pressure. Therefore the separation times shown in Figure 9 represent the lowest that can be obtained from settling alone. Bulk temperature measurements after microwave heating indicate that the emulsion temperature is of the order of $40-50^{\circ} \mathrm{C}$, so the data in Figure 9 represent a conservative estimate of the minimum separation time that can be achieved. The experimentally observed settling time is still much lower than that predicted by the model, indicating that the average droplet sizes must be larger than $345 \mu \mathrm{m}$.

The predictions shown in Figures 8 and 9 show that the enhanced separation of emulsions due to microwave heating cannot be solely attributed to a viscosity reduction in the oil phase - significant coalescence of the water droplets must also occur during the heating and settling process. The localised temperature of the oil adjacent to the water droplets is not known, therefore it is not possible to completely decouple the effects of coalescence and viscosity from the data presented in this study. However future work in this area will aim to quantify the two effects using insitu droplet size measurement techniques along with more sophisticated CFD modelling approaches to assess thermal and viscosity gradients throughout the emulsion.

The results of this work can be used to elucidate the mechanism of the well-known phenomenon of microwave enhanced settling of water-in-oil emulsions. While the complexity of the system has prevented complete decoupling of the effects of 
viscosity and coalescence on settling time, it is possible to explain both factors by the thermal effects of microwave heating. The water phase is heated selectively, so the temperature of the oil at the droplet interface is much higher than the bulk oil temperature. A temperature gradient exists from the bulk oil to the droplet interface, which is unique to microwave heating. The high interface temperature results in a lower oil viscosity, but also a reduced interfacial tension $[1,2]$. Interfacial tension is reduced further due to the volumetric expansion of the water droplet resulting from its selective heating. Coalescence can be increased by increasing the collision frequency between droplets, and by decreasing the resistance to coalescence. A lower viscosity at the interface increases the collision frequency as there is less resistance to droplet flow. A lower interfacial tension means that resistance to coalescence is reduced when droplets collide, therefore improving the probability of coalescence.

The improvements in separation time observed with microwave heating of salinebased emulsions compared with water-based emulsions can also be explained in terms of thermal phenomenon that arise from microwave heating. The evidence presented in Figures 4 and 7 leads to the conclusion that the predominant effect of the addition of $\mathrm{NaCl}$ is to enhance the thermal effect during microwave heating, rather than directly changing the interfacial tension.

\section{CONCLUSIONS}

This study has shown categorically that microwave heating results in enhanced droplet coalescence of the water phase in water-in-oil emulsions. Microwave heating results in an order of magnitude improvement in separation time, with the lowest 
heating times occurring with saline emulsions. Saline emulsions separate more rapidly due to the change in dielectric properties and the change in interfacial tension, which promotes a larger thermal gradient and improved probability of coalescence. The improvements in settling rate can all be rationalised by the unique thermal gradients that arise from microwave heating, and their subsequent impact on viscosity and interfacial tension at the droplet/oil interface. Contrary to other reports in the literature, no evidence for non-thermal effects was observed in this study.

\section{ACKNOWLEDGEMENT}

The authors wish to acknowledge BP for funding this work.

\section{REFERENCES}

[1] K. Arnold, M. Stewart, Surface Production Operations - Design of Oil Handling Systems and Facilities, 3rd Edition ed., Elsevier, 1998.

[2] K.S. Birdi, Handbook of surface and colloid chemistry, CRC Press, 2009.

[3] C. Holtze, R. Sivaramakrishnan, M. Antonietti, J. Tsuwi, F. Kremer, K.D. Kramer, The microwave absorption of emulsions containing aqueous micro- and nanodroplets: A means to optimize microwave heating, Journal of Colloid and Interface Science, 302 (2006) 651-657.

[4] M. Fortuny, C.B.Z. Oliveira, R.L.F.V. Melo, M. Nele, R.C.C. Coutinho, A.F. Santos, Effect of salinity, temperature, water content, and $\mathrm{pH}$ on the microwave demulsification of crude oil emulsions, Energy and Fuels, 21 (2007) 1358-1364. [5] C.S. Fang, P.M.C. Lai, B.K.L. Chang, W.J. Klaila, Oil recovery and waste reduction by microwave radiation, Environmental Progress, 8 (1989) 235-238. 
[6] L. Xia, S. Lu, G. Cao, Demulsification of Emulsions Exploited by Enhanced Oil Recovery System, Separation Science and Technology, 38 (2003) 4079-4094.

[7] L.X. Xia, S.W. Lu, G.Y. Cao, Salt-assisted microwave demulsification, Chemical Engineering Communications, 191 (2004) 1053-1063.

[8] B.M.S. Ferreira, J.B.V.S. Ramalho, E.F. Lucas, Demulsification of eater-in-crude oil emulsions by microwave radiation: Effect of aging, demulsifier addition, and selective heating, Energy and Fuels, 27 (2013) 615-621.

[9] C.S. Fang, P.M.C. Lai, Microwave heating and separation of water-in-oil emulsions, Journal of Microwave Power and Electromagnetic Energy, 30 (1995) 4657.

[10] W. Tan, X.G. Yang, X.F. Tan, Study on demulsification of crude oil emulsions by microwave chemical method, Separation Science and Technology, 42 (2007) 1367-1377.

[11] R.C.B. Lemos, E.B. Da Silva, A. Dos Santos, R.C.L. Guimarães, B.M.S. Ferreira, R.A. Guarnieri, C. Dariva, E. Franceschi, A.F. Santos, M. Fortuny, Demulsification of water-in-crude oil emulsions using ionic liquids and microwave irradiation, Energy and Fuels, 24 (2010) 4439-4444.

[12] D. Guzmán-Lucero, P. Flores, T. Rojo, R. Martínez-Palou, Ionic liquids as demulsifiers of water-in-crude oil emulsions: Study of the microwave effect, Energy and Fuels, 24 (2010) 3610-3615.

[13] M. Fortuny, E.B. Silva, A.C. Filho, R.L.F.V. Melo, M. Nele, R.C.C. Coutinho, A.F. Santos, Measuring Salinity in crude oils: Evaluation of methods and an improved procedure, Fuel, 87 (2008) 1241-1248. 
[14] E.R. Binner, J.R. Robinson, S.W. Kingman, E.H. Lester, B.J. Azzopardi, G. Dimitrakis, J. Briggs, Separation of oil/water emulsions in continuous flow using microwave heating, Energy and Fuels, (in press).

[15] A.H. Nour, R.M. Yunus, A comparative study on emulsion demulsification by microwave radiation and conventional heating, Journal of Applied Sciences, 6 (2006) 2307-2311.

[16] L.A. Kovaleva, R.Z. Minnigalimov, R.R. Zinnatullin, Destruction of water-in-oil emulsions in radio-frequency and microwave electromagnetic fields, Energy and Fuels, 25 (2011) 3731-3738.

[17] Azeri (Ceyhan) Assay, in.

[18] R.J. Meredith, Engineers Handbook of Industrial Microwave Heating, IEEE, London, 1998. 


\section{Figures with captions}

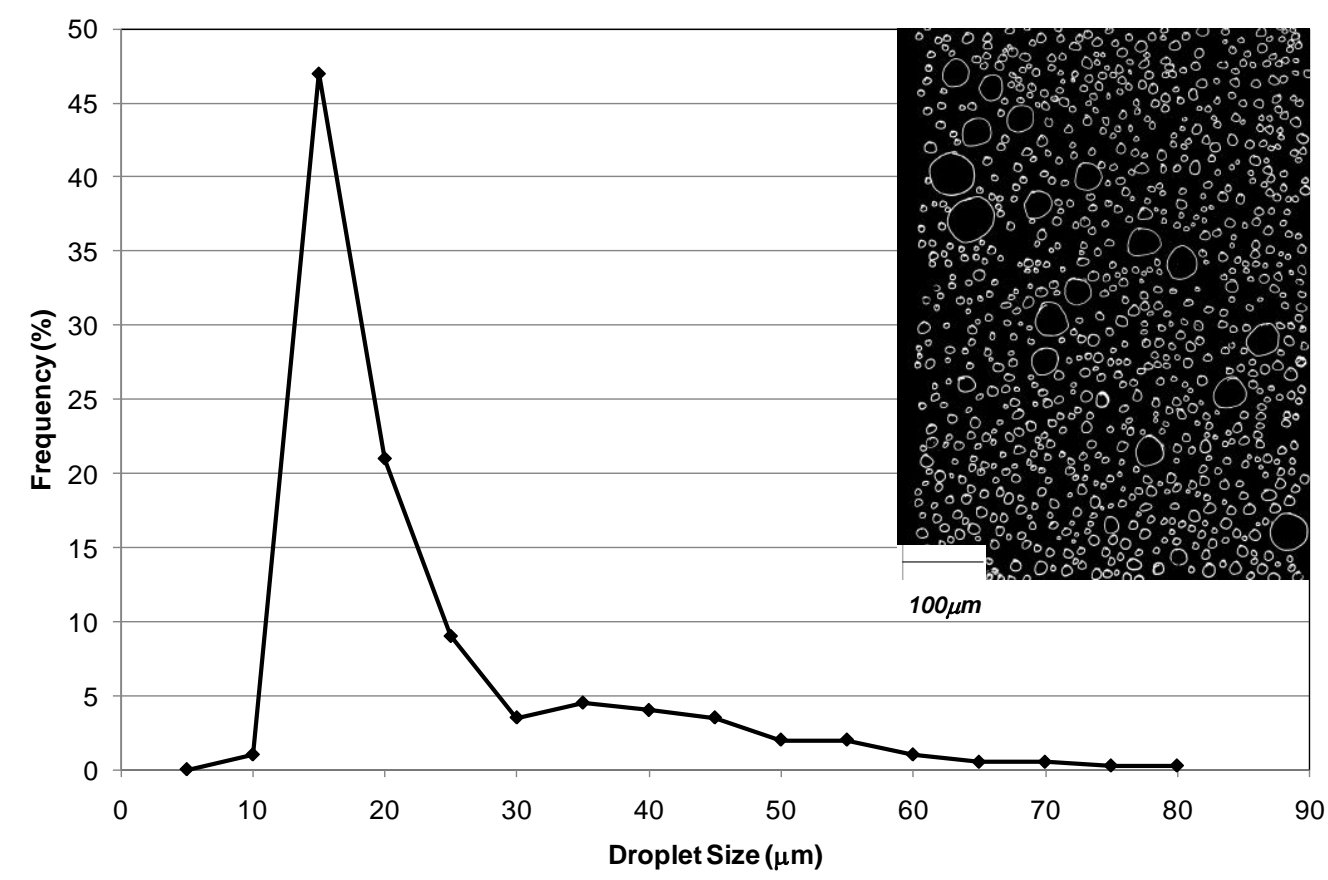

Figure 1. Size distribution of water droplets formed during emulsification for $30 \%$ water. Inset image shows the water droplets after thresholding from the background oil using image analysis [14].

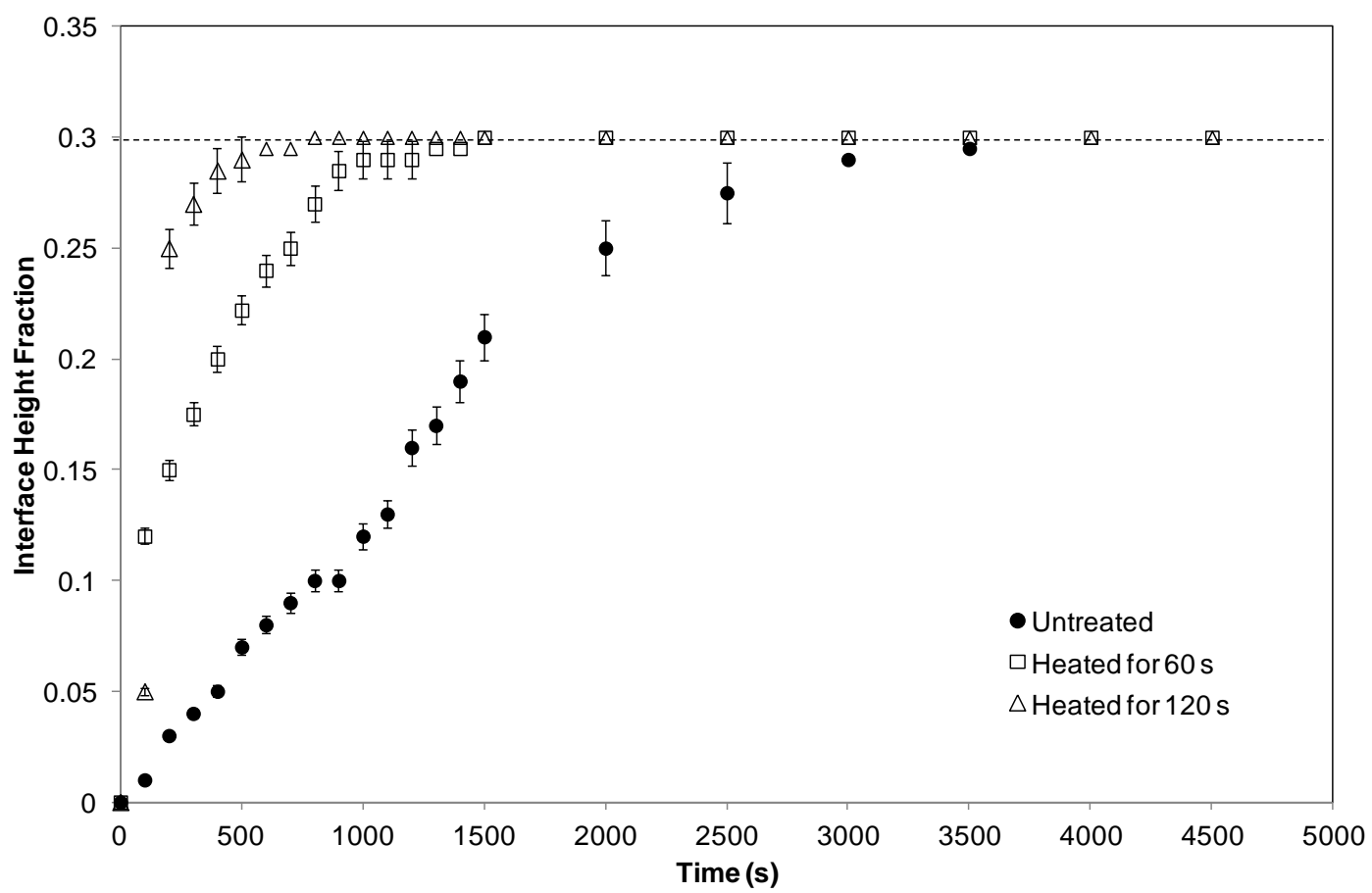

Figure 2. Oil/water interface height plotted against time for a 70/30 oil/water emulsion under different heating regimes 


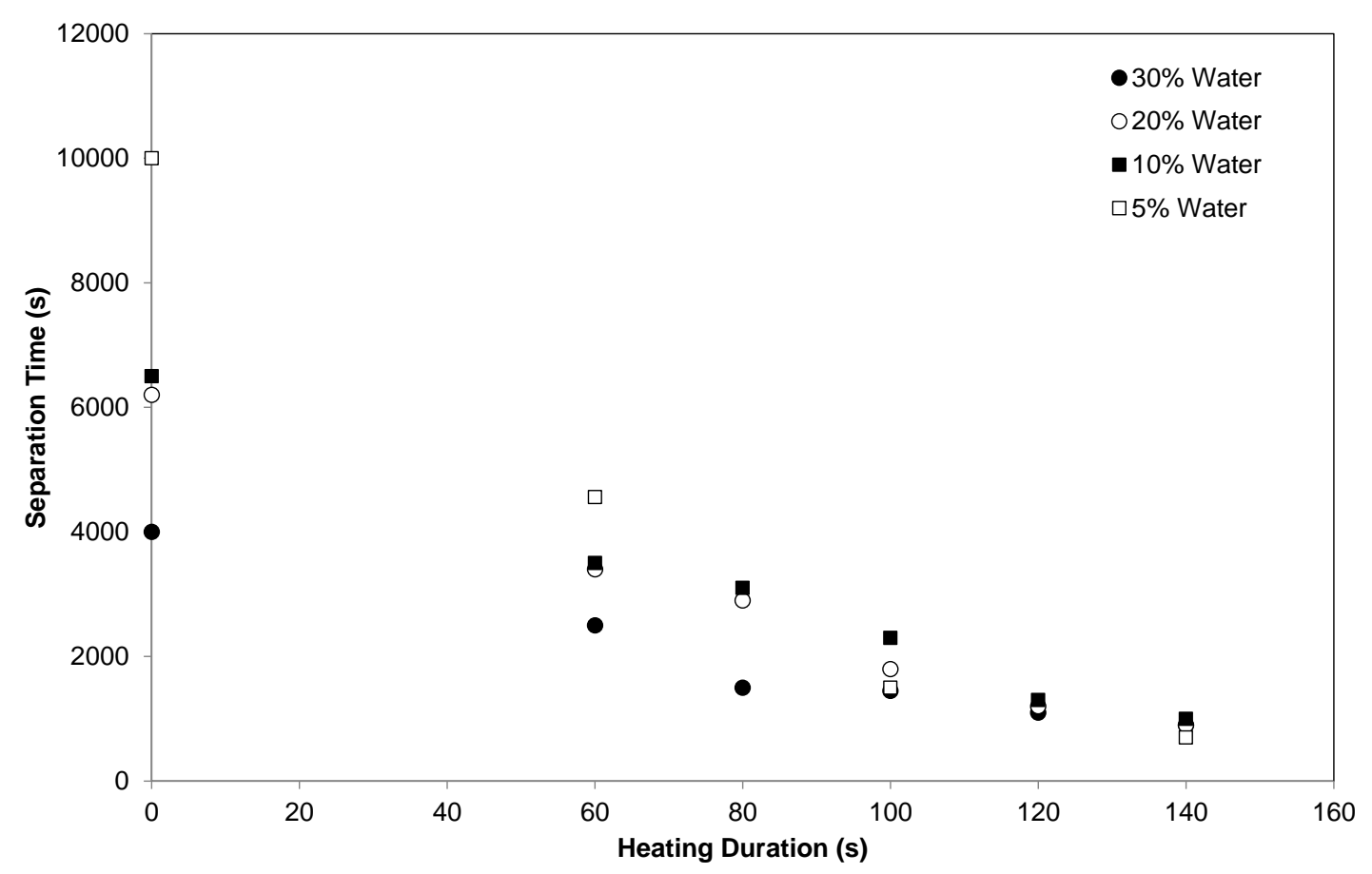

Figure 3. Effect of water content on separation time for a range of microwave heating times

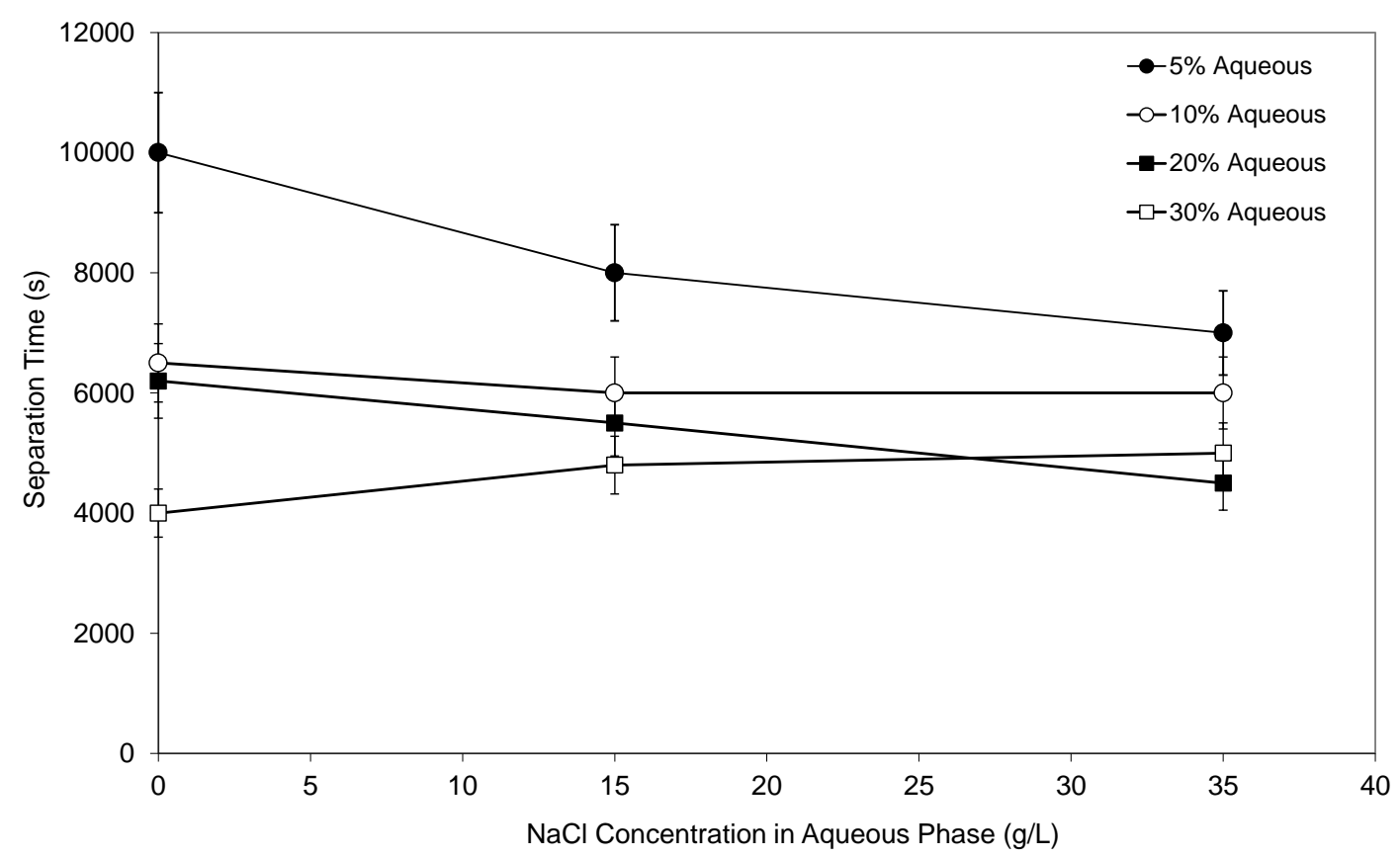

Figure 4. Effect of salt concentration on separation time without the influence of microwave heating 


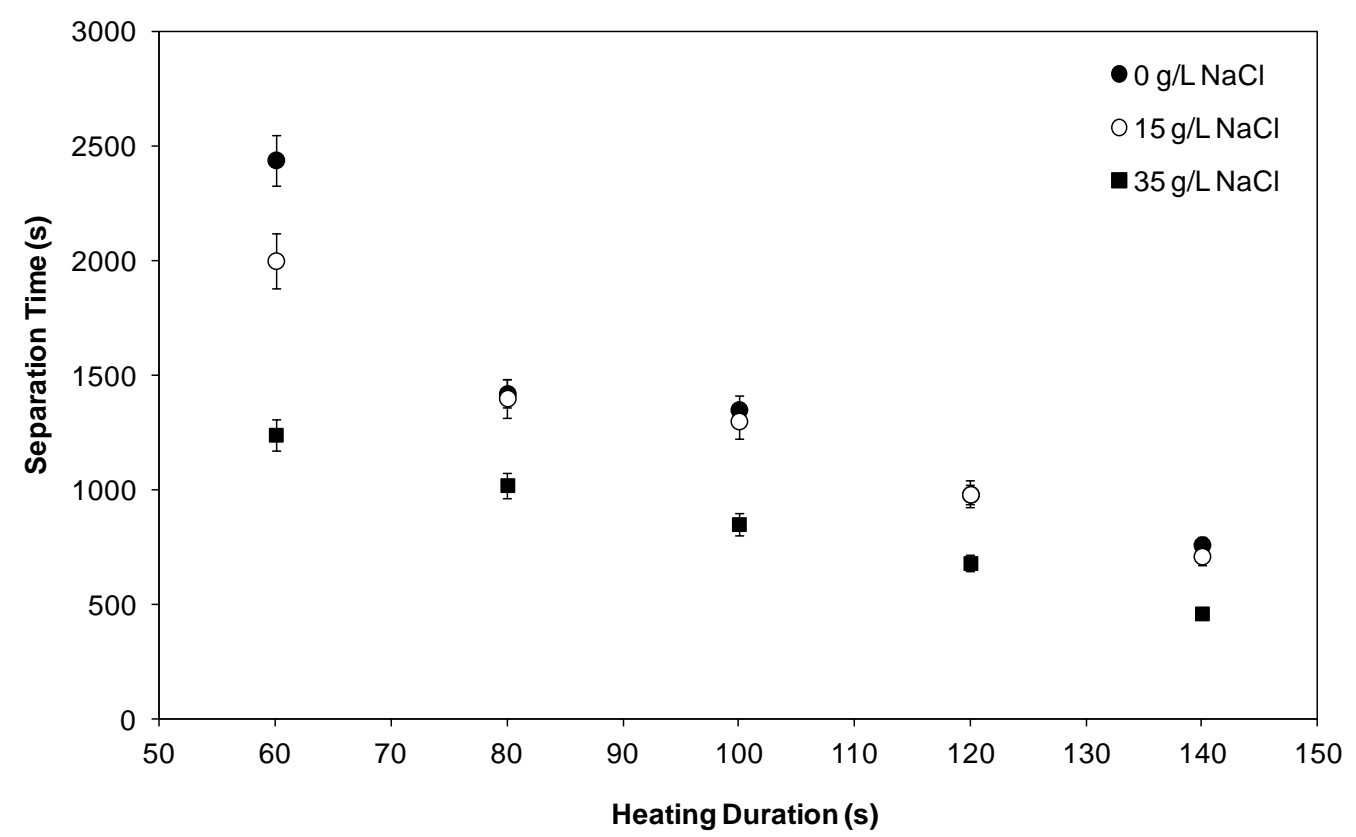

Figure 5. Effect of $\mathrm{NaCl}$ concentration on the separation of 70/30 emulsions during microwave heating

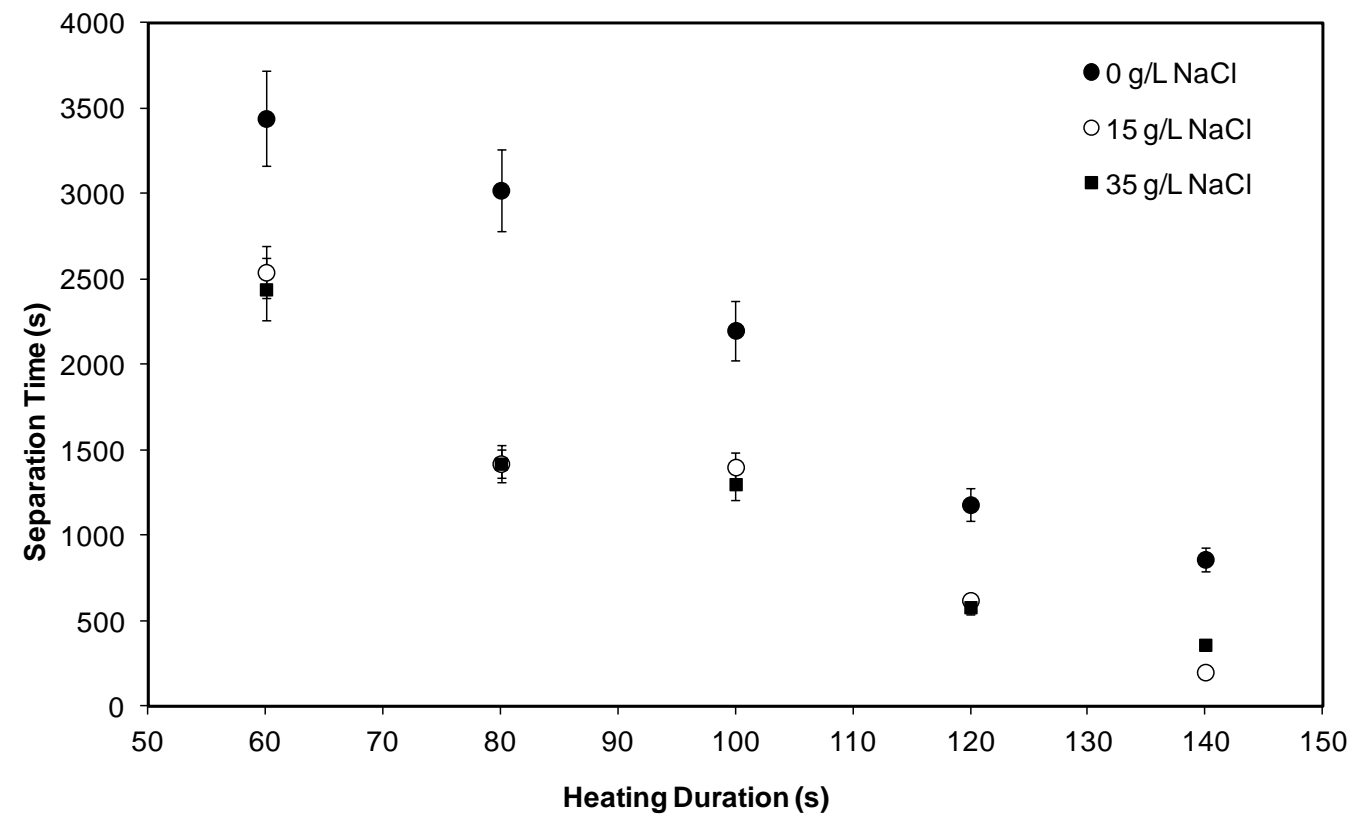

Figure 6. Effect of $\mathrm{NaCl}$ on the separation of 90/10 emulsions during microwave heating 

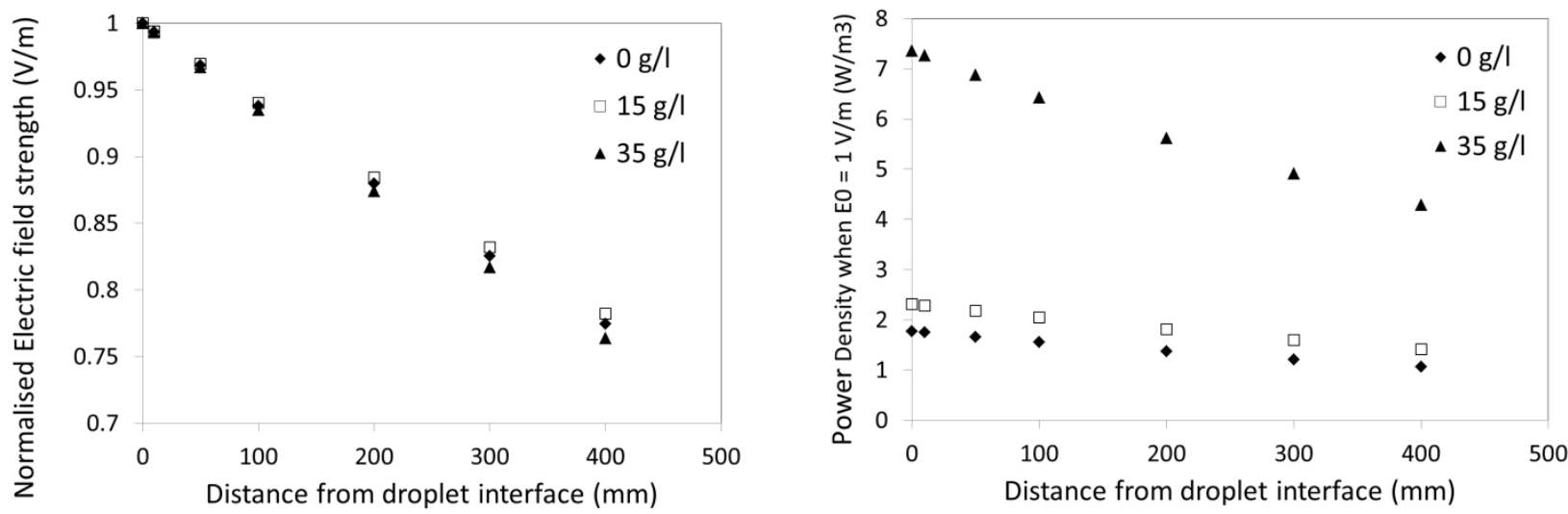

Figure 7. Relative electric field (left) and power density (right) distribution within water droplets of varying salinity

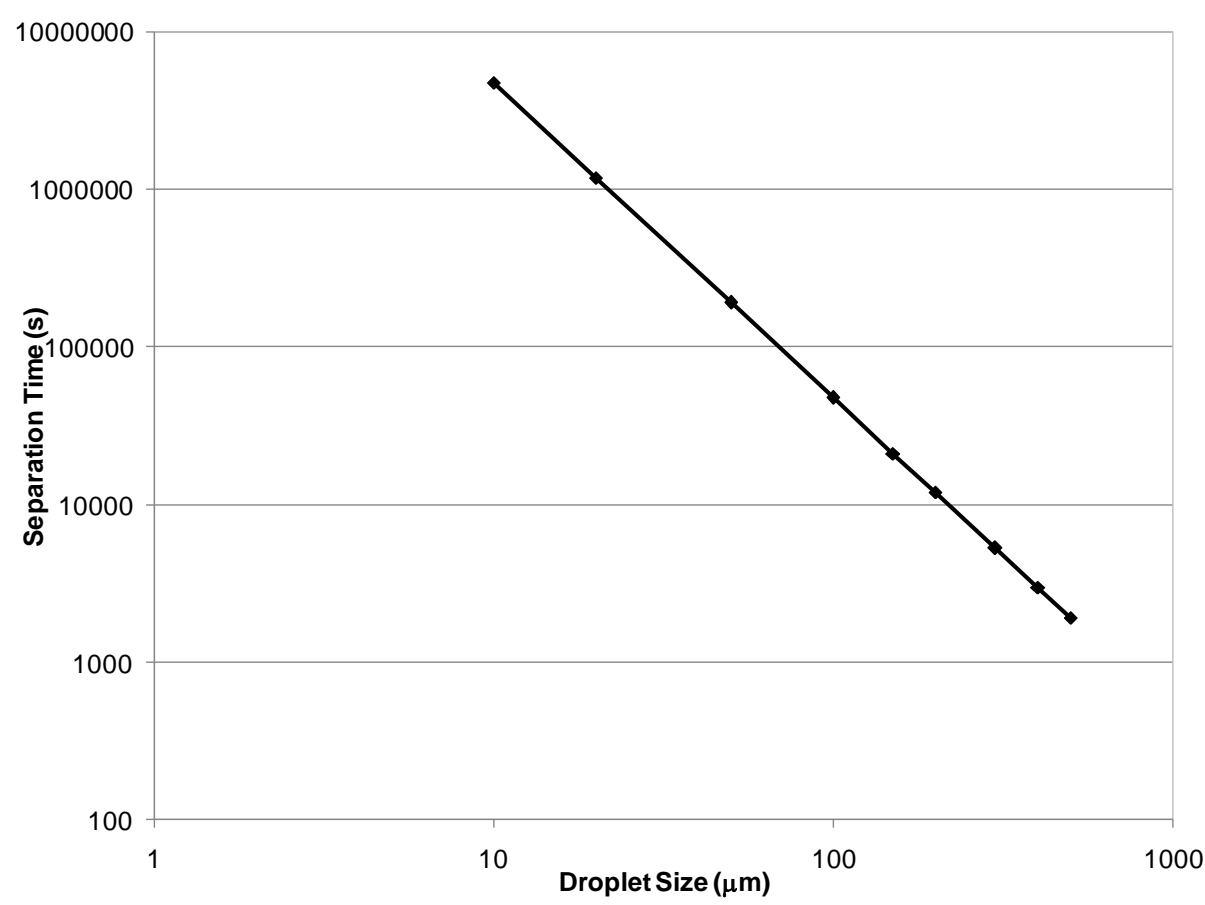

Figure 8. Predicted separation time at $20^{\circ} \mathrm{C}$ plotted against droplet size. Model assumes settling only, with no coalescence. 


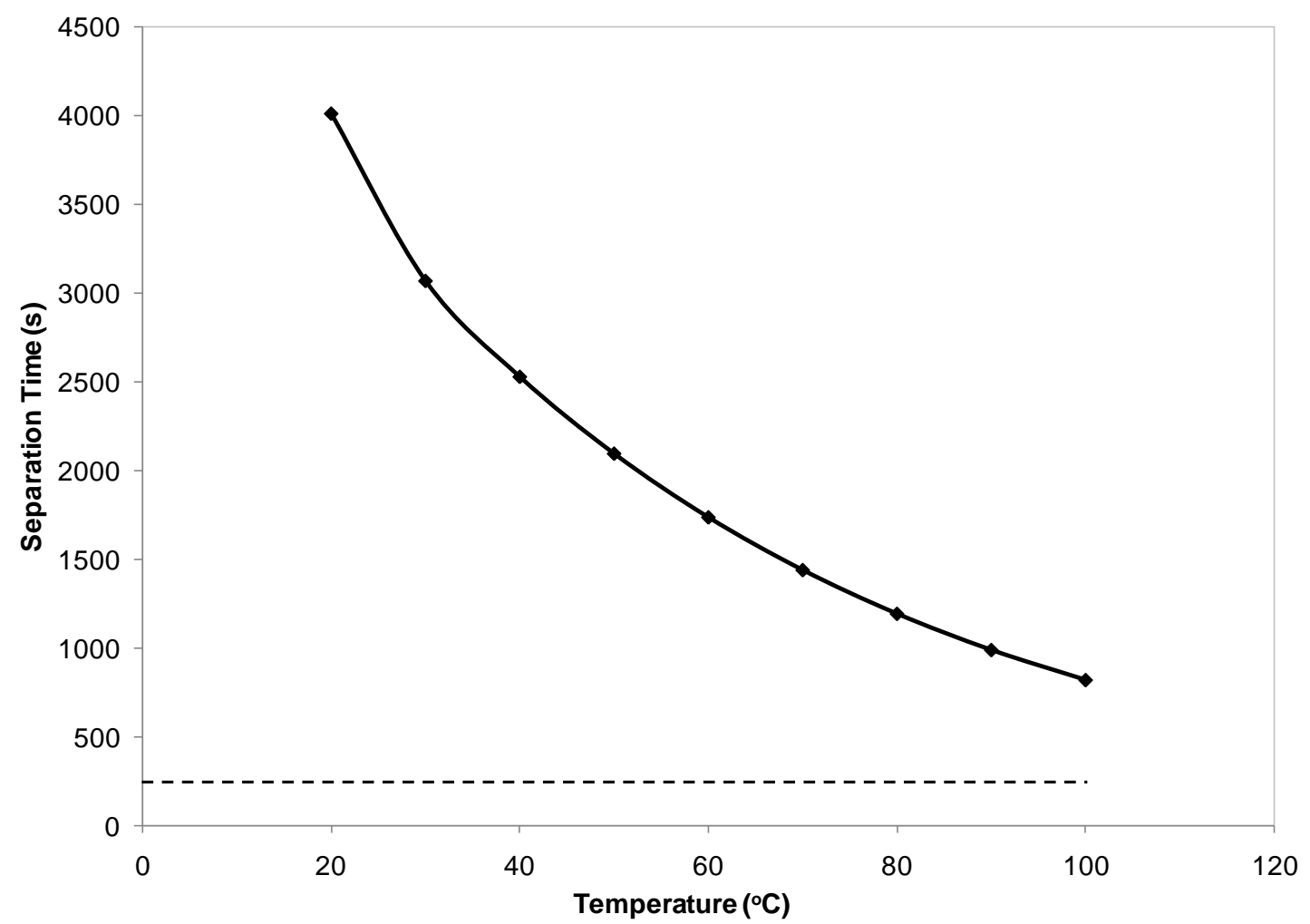

Figure 9. Theoretical separation time plotted against oil temperature for a mean droplet diameter of $345 \mu \mathrm{m}$. Dashed line shows the minimum experimentally-observed separation time. 